\title{
Enhancing the Performance of Quantum Dot Light-Emitting Diodes Using Room-Temperature-Processed Ga-Doped ZnO Nanoparticles as the Electron Transport Layer
}

\author{
Sheng Cao, ${ }^{\dagger, \ddagger}, \square$ Jinju Zheng, ${ }^{\dagger, \square}$ Jialong Zhao, ${ }^{\S}$ Zuobao Yang, ${ }^{\dagger}$ Chengming Li, ${ }^{\ddagger}$ Xinwei Guan, ${ }^{\#}$ Weiyou \\ Yang, ${ }^{\dagger}$ Minghui Shang, ${ }^{* \dagger}$ and Tom Wu, \\ ${ }^{\dagger}$ Institute of Materials, Ningbo University of Technology, Ningbo 315016, China. \\ ${ }^{\ddagger}$ Institute for Advanced Materials and Technology, University of Science and Technology Beijing, Beijing 100083, China. \\ ${ }^{\S}$ Key Laboratory of Functional Materials Physics and Chemistry of the Ministry of Education, Jilin Normal University, Siping \\ 136000, China \\ " Materials Science and Engineering, King Abdullah University of Science and Technology (KAUST), Thuwal 23955-6900, \\ Saudi Arabia
}

KEYWORDS: LED, Ga-doped ZnO, Nanoparticles, Electron transport layer, Charge transfer

\begin{abstract}
Colloidal $\mathrm{ZnO}$ nanoparticle (NP) films are recognized as efficient electron transport layers (ETLs) for quantum dot light emitting diodes (QD-LEDs) with good stability and high efficiency. However, because of the inherently high work function of such films, spontaneous charge transfer occurs at the QD/ZnO interface in such a QD-LED, thus leading to reduced performance. Here, to improve the QD-LED performance, we prepared Ga-doped ZnO NPs with low work functions and tailored band structures via a room-temperature (RT) solution process without the use of bulky organic ligands. We found that the charge transfer at the interface between the CdSe/ZnS QDs and the doped $\mathrm{ZnO}$ NPs was significantly weakened because of the incorporated Ga dopants. Remarkably, the as-assembled QD-LEDs with Ga doped ZnO NPs as the ETLs exhibited superior luminances of up to $44000 \mathrm{~cd} / \mathrm{m} 2$ and efficiencies of up to $15 \mathrm{~cd} / \mathrm{A}$, placing them among themost efficient red-light QD-LEDs ever reported. This discovery provides a new strategy for fabricating high-performance QD-LEDs by using RT-processed Ga-doped ZnO NPs as the ETLs, which could be generalized to improve the efficiency of other optoelectronic devices.
\end{abstract}

\section{INTRODUCTION}

In recent years, quantum dot light-emitting diodes (QD-LEDs) have received considerable attention in both academia and industry for their potential application in next-generation low-cost and highefficiency flat panel displays and solid-state lighting by virtue of their unique properties, such as tunable emission wavelengths, highly saturated emission with small full widths at half maximum (FWHMs), solution processability, and compatibility with flexible substrates. ${ }^{1-11}$ Pioneering work on multilayer QD-LEDs was performed in the early 1990s. ${ }^{1}$ Soon after this breakthrough, enormous efforts were directed toward synthesizing qualified QDs, understanding the fundamental physics, and developing suitable device architectures. ${ }^{9,}{ }^{12-17} \mathrm{In}$ particular, the use of $\mathrm{ZnO}$ nanoparticle (NP) films as the electron transport layers (ETLs) in QD-LEDs was introduced in 2011 by Qian et al. and shown to significantly enhance the environmental stability and efficiency of the resulting devices. ${ }^{11}$ Furthermore, the realization of $\mathrm{ZnO}$ NP inks based on colloids dispersed in suitable solvents could offer great advantages for many applications, considering that the relevant wet deposition techniques (e.g., spraying, inkjet/gravure printing, spinning, and so forth) permit the coating of a broad range of substrates and shapes at a reasonable cost. Consequently, $\mathrm{ZnO}$ NPs have come to be recognized as the primary candidate electron transport material for the construction of highly bright and efficient QD-LEDs. ${ }^{2,6,9,12,18}$

However, similarly to other metal oxides with high work functions, $\mathrm{ZnO}$ NPs quench the photoluminescence (PL) quantum yields (QYs) of emitting QDs by means of spontaneous charge transfer occurring at the $\mathrm{QD} / \mathrm{ZnO}$ interface, thus leading to performance degradation in QD-LEDs. ${ }^{2,7-8,18-21}$ To date, three typical strategies have been developed to overcome this intrinsic shortcoming: (i) insert a thin insulating layer (e.g., poly(methyl methacrylate) (PMMA)) to modify the $\mathrm{QD} / \mathrm{ZnO}$ interfacial interaction, ${ }^{2}$ (ii) deposit a thin semiconducting film on the $\mathrm{ZnO}$ layer (e.g., polyethylenimine ethoxylated (PEIE) or cesium carbonate $\left(\mathrm{CsCO}_{3}\right)$ ) to establish a low work function to limit the charge transfer, ${ }^{17,22-23}$ or (iii) tailor the energy structure of the $\mathrm{ZnO}$ (e.g., through size control, doping, and surface modification) to lower its work function. ${ }^{18,24-25}$ Among these possibilities, the third one, namely, modifying the energy structure of the $\mathrm{ZnO}$ ETL itself, is an effective and low-cost method for enabling the fabrication of highly bright and efficient QD-LEDs. 
To this end, modifying the energy structure of $\mathrm{ZnO}$ via doping is considered an effective technique for tailoring its electronic structure. ${ }^{26}$ Based on this approach, highly qualified colloidal Mg-, In-, Al- and Ga-doped ZnO NPs with controllable sizes and shapes as well as high crystallinity have been reported. ${ }^{25,27-34}$ Compared with group II elements such as $\mathrm{Mg},{ }^{35-36}$ their group III counterparts, such as the n-type dopants $\mathrm{Ga}$, In and $\mathrm{Al}$, can often raise the Fermi levels of their $\mathrm{ZnO}$ hosts. Furthermore, it is noted that the covalent bond length of Ga-O (1.92 Å) is similar to that of $\mathrm{Zn}-\mathrm{O}(1.97 \AA) ;{ }^{37}$ in addition, $\mathrm{Ga}$ ions are also similar in electronegativity and ion radius to the $\mathrm{Zn}$ ions in the $\mathrm{ZnO}$ host and therefore are expected to be readily incorporated into $\mathrm{ZnO}$ with limited crystal defects. ${ }^{29,31,33,38-}$

${ }^{39}$ Regarding state-of-the-art Ga-doped $\mathrm{ZnO} \mathrm{NPs}$, although a few groups have successfully grown Ga-doped $\mathrm{ZnO}$ NPs with controlled carrier concentrations, ${ }^{28,31,33}$ most of the implemented procedures have relied on high-temperature approaches and have required the use of bulky organic ligands, leading to poor electrical conductivity of the as-grown $\mathrm{ZnO} \mathrm{NPs}$. Consequently, either tedious ligand exchanges or high post-annealing temperatures of greater than $200^{\circ} \mathrm{C}$ and UV irradiation have been necessary to remove the insulating ligands to achieve the desired high conductivity. ${ }^{28,31,40}$ However, such high-temperature annealing may pose considerable problems for $\mathrm{ZnO}$ NPs that are to serve as the ETLs in QD-LEDs because of the inevitable thermal degradation of the polymer hole transport layer and the QD emitting layer. Therefore, a facile method of growing Ga-doped ZnO NPs without the need for bulky organic ligands or post-treatments is a critical requirement for exploring the fabrication of high-performance QD-LEDs.

Herein, for the first time, we report the controlled growth of colloidal Ga-doped ZnO NPs via a low-temperature hydrolysis process without bulky organic ligands. By varying the nominal Ga content between 0 and 12 at.\%, highly monodisperse Ga-doped ZnO NPs were successfully synthesized. Remarkably, NP films with high conductivity and improved surface flatness could be easily obtained via spin coating at room temperature (RT) without any post-treatment. The time-resolved PL dynamics confirmed that the charge transfer process at the $\mathrm{QD} /$ doped $\mathrm{ZnO}$ interfaces was profoundly weakened by the Ga dopants; this finding is ascribed to the increase in the Fermi level of the $\mathrm{ZnO} N P s$ induced by the Ga dopants. Asassembled QD-LEDs with ETLs consisting of Ga-doped ZnO NPs exhibited a superior luminance of $44000 \mathrm{~cd} / \mathrm{m}^{2}$ and a mean current efficiency of $13.1 \mathrm{~cd} / \mathrm{A}$ (with a maximum efficiency of $15 \mathrm{~cd} / \mathrm{A}$ ), which is comparable to the highest efficiency value ever reported, suggesting their great potential for application in high-performance optoelectronics.

\section{RESULTS AND DISCUSSION}

Synthesis and Characterization of Ga-doped ZnO NPs To date, qualified monodisperse Ga-doped ZnO NPs with controlled sizes, shapes, and crystallinity have typically been synthesized using hightemperature approaches. ${ }^{28,31,34}$ Notably, such high-temperature methods often require bulky organic ligands, which lead to poor electrical conductivity of the as-synthesized NPs and consequently hinder their subsequent device applications. Distinct from these previously reported works, we developed a facile synthesis strategy for preparing high-quality colloidal Ga-doped $\mathrm{ZnO}$ NPs via the hydrolysis of zinc salts at low temperatures without the need for bulky organic ligands or post-treatments.

Table 1 shows the chemical compositions of Ga-doped ZnO NPs with different nominal doping levels as measured by inductively coupled plasma optical emission spectrometry (ICP-OES). As shown in the table, when a higher molar ratio of gallium acetate was intro- duced into the raw materials, a higher doping concentration was achieved in the obtained NPs. However, it was found that the real Ga doping levels were slightly higher than the nominal ones, which might be attributable to the different chemical reactivities of the $\mathrm{Zn}$ and Ga precursors.

Figure 1a presents the typical X-ray diffraction (XRD) patterns of Ga-doped ZnO NPs with different nominal Ga doping levels. As shown in Figure 1a, all samples exhibited an archetypal wurtzitetype $\mathrm{ZnO}$ structure, and the diffraction peaks clearly deviated from those of $\mathrm{Ga}_{2} \mathrm{O}_{3}$, indicating that the products contained no $\mathrm{Ga}_{2} \mathrm{O}_{3}{ }^{41}$ In addition, the reflection peaks became increasingly broad with a higher Ga doping level. According to the Debye-Scherrer equation, their crystallite sizes were approximately 4.4, 4.2, 4.1, 4.0, 3.9 and $3.8 \mathrm{~nm}$ for nominal Ga doping levels of 0, 1, 2, 4, 8 and 12 at.\%, respectively. Their calculated lattice parameters are summarized in Table S1 (Supporting Information). The slight deviations of the lattice parameters compared with those of pure $\mathrm{ZnO}$ can be understood based on a comparison of the ionic radii of $\mathrm{Zn}^{2+}(0.74 \AA)$ and $\mathrm{Ga}^{3+}(0.62 \AA)$ as well as the lengths of covalent Ga-O (1.92 $\AA$ ) and Zn-O (1.97 Å) bonds; ${ }^{24,33,37,42-43}$ this comparison indicates that the incorporation of $\mathrm{Ga}^{3+}$ dopants into the $\mathrm{ZnO}$ NPs mainly occurred through the formation of substitutional solid solutions (schematically illustrated in Figure 1b). ${ }^{44}$

Table 1. Chemical compositions of Ga-doped $\mathrm{ZnO}$ NPs with different doping levels as measured by ICP-OES. In general, the actual doping levels are higher than the nominal ones.

\begin{tabular}{llll}
\hline Sample & $\begin{array}{l}\text { Nominal } \\
\text { [Ga] (at.\%) }\end{array}$ & $\begin{array}{l}\text { [Ga] in NPs } \\
\text { (at.\%) }\end{array}$ & $\begin{array}{l}\text { [Zn] in NPs } \\
\text { (at.\%) }\end{array}$ \\
\hline S0 & 0 & 0 & 100 \\
S1 & 1 & 1.2 & 98.8 \\
S2 & 2 & 2.6 & 97.4 \\
S4 & 4 & 4.5 & 95.5 \\
S8 & 8 & 11.6 & 88.4 \\
S12 & 12 & 16.5 & 83.5 \\
\hline
\end{tabular}

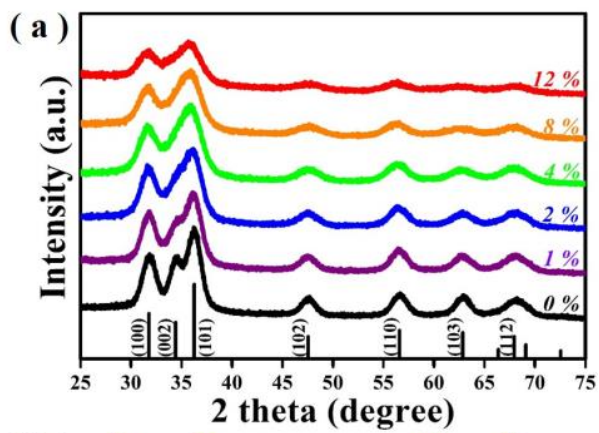

( b )

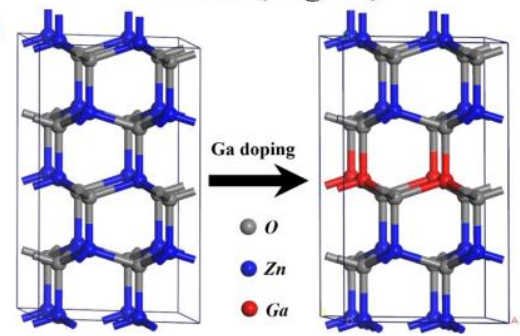

Figure 1. (a) XRD patterns of Ga-doped ZnO NPs with different nominal doping levels. These $\mathrm{ZnO}$ NPs were prepared via the roomtemperature hydrolysis of $\mathrm{Zn}$ salts in basic solutions. (b) Schematic illustration of the $\mathrm{Ga}$ doping of the $\mathrm{ZnO}$ crystal lattice via the formation of substitutional solid solutions. 

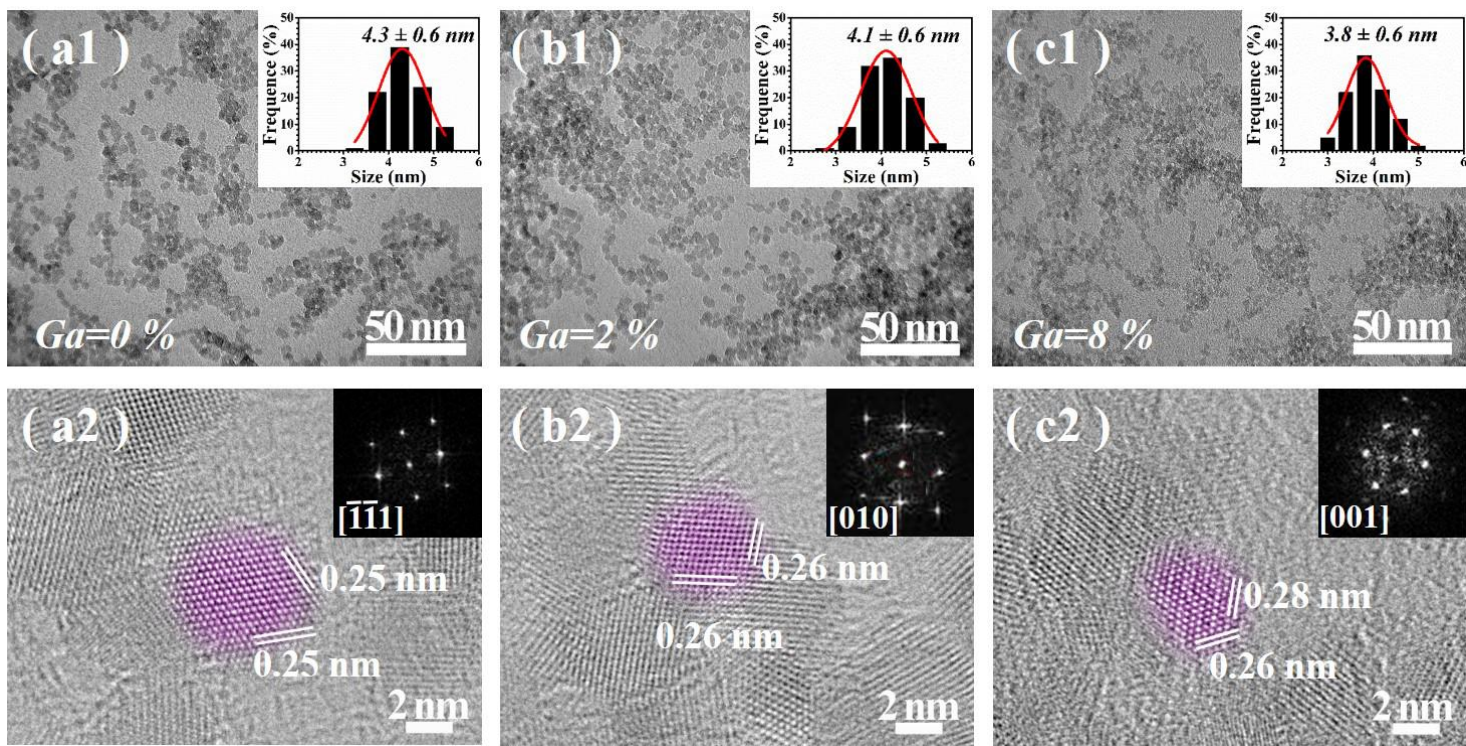

Figure 2. (a1, b1, c1) TEM images and (a2, b2, c2) HRTEM images of Ga-doped ZnO NPs from Samples S0, S2 and S8, respectively. The insets in $(\mathrm{a} 1, \mathrm{~b} 1, \mathrm{c} 1)$ show the statistical size distributions, and those in $(\mathrm{a} 2, \mathrm{~b} 2, \mathrm{c} 2)$ present the FFT patterns of the corresponding samples.
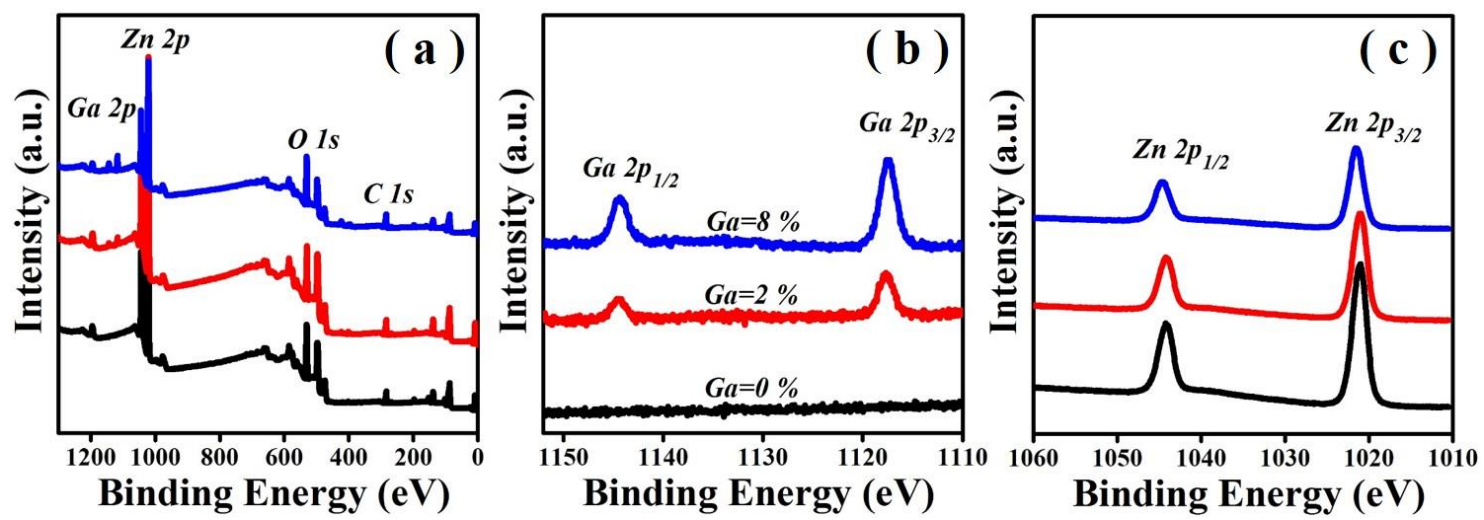

Figure 3. (a) XPS survey peaks, (b) Ga 2p core-level peaks, and (c) Zn 2p core-level peaks of Ga-doped ZnO NPs from Samples SO (black lines), S2 (red lines) and S8 (blue lines).

Figure 2 shows representative transmission electron microscopy (TEM) and high-resolution TEM (HRTEM) images of Ga-doped $\mathrm{ZnO}$ NPs from Samples S0, S2 and S8 (i.e., with nominal doping levels of 0,2 and 8 at.\%). As shown in Figures 2a1-c1, all of the doped $\mathrm{ZnO}$ samples were monodisperse, with narrow diameter distributions of $4.3 \pm 0.6,4.1 \pm 0.6$ and $3.8 \pm 0.6 \mathrm{~nm}$ for Samples SO, S2 and S8, respectively. It seems that a higher Ga doping level resulted in smaller NPs, which is consistent with the broadened diffraction peaks in the XRD profiles at higher Ga doping levels (Figure 1a). Figures 2a2-c2 show typical HRTEM images and the corresponding fast Fourier transform (FFT) patterns (the insets in Figures 2a2-c2, respectively) for single NPs, which reveal their singlecrystalline nature. The well-resolved lattice fringes with interplanar spacings of $0.25,0.26$ and $0.28 \mathrm{~nm}$ correspond to the (100), (002) and (100) lattice planes, respectively, of wurtzite $\mathrm{ZnO} .^{30,36,41,45} \mathrm{No}$ tably, no particle with interplanar spacings close to those of corundum-type $\mathrm{Ga}_{2} \mathrm{O}_{3}$ was found, further confirming that the $\mathrm{Ga}$ was incorporated into the $\mathrm{ZnO}$ matrix without the formation of $\mathrm{Ga}_{2} \mathrm{O}_{3}$.
Figure 3 shows representative X-ray photoelectron spectroscopy (XPS) spectra of the Ga-doped ZnO NPs from Samples S0, S2 and $\mathrm{S} 8$, which reveal that elements of $\mathrm{Ga}, \mathrm{Zn}$, and $\mathrm{O}$ existed in the products, whereas the pure $\mathrm{ZnO} \mathrm{NP}$ sample was composed only of $\mathrm{Zn}$ and $\mathrm{O}$ elements. The $\mathrm{C}$ signal is attributed to adventitious carbon, and the peak positions were calibrated by using the $\mathrm{C} 1 \mathrm{~s}$ peak at $284.6 \mathrm{eV}$ as a reference. Figures $3 \mathrm{~b}$ and $3 \mathrm{c}$ show the highresolution Ga $2 \mathrm{p}$ and $\mathrm{Zn} 2 \mathrm{p}$ XPS spectra, respectively. For the $\mathrm{ZnO}$ NPs with a nominal Ga doping level of 2 at.\% (Figure 3b), the two peaks detected at 1144.58 and $1117.68 \mathrm{eV}$ are attributed to the Ga $2 \mathrm{p}_{1 / 2}$ and Ga $2 \mathrm{p}_{3 / 2}$ core levels, respectively. ${ }^{36,46}$ The $2 \mathrm{p}_{3 / 2}$ core level of $\mathrm{Ga}(1117.68 \mathrm{eV})$ shows a positive shift relative to that of elemental $\mathrm{Ga}\left(\mathrm{Ga} 2 \mathrm{p}_{3 / 2}, 1116.67 \mathrm{eV}\right)$, indicating that the $\mathrm{Ga}$ was doped into the $\mathrm{ZnO}$ crystal lattice in a compound state. The peaks at $\sim 1044$ and $\sim 1021 \mathrm{eV}$ in the $\mathrm{Zn} 2 \mathrm{p}$ spectra (Figure 3c) of the three samples are assigned to $\mathrm{Zn} 2 \mathrm{p}_{1 / 2}$ and $\mathrm{Zn} 2 \mathrm{p}_{3 / 2}$, respectively, suggesting that they are characteristic of $\mathrm{Zn}-\mathrm{O}$ bonds. ${ }^{44}$ Interestingly, unlike the $\mathrm{Zn} 2 \mathrm{p}$ peaks, which exhibited a shift toward higher binding energies with an increasing doping level (i.e., from 1044.11 to 
$1044.58 \mathrm{eV}$ for $\mathrm{Zn} 2 \mathrm{p}_{1 / 2}$ and from 1021.07 to $1021.48 \mathrm{eV}$ for $\mathrm{Zn}$ $2 p_{3 / 2}$ ), the $G a 2 p$ peaks showed a different trend (i.e., shifting from 1044.58 to $1044.13 \mathrm{eV}$ for $\mathrm{Ga} 2 \mathrm{p}_{1 / 2}$ and from 1017.68 to 1017.28 $\mathrm{eV}$ for $\mathrm{Ga} 2 \mathrm{p}_{3 / 2}$ ), which can be attributed to the different electronegativities of $\mathrm{Ga}(1.81)$ and $\mathrm{Zn}$ (1.65). These results confirm that the Ga dopants were successfully incorporated into the $\mathrm{ZnO}$. They also suggest that $\mathrm{Ga}$ ions incorporated into $\mathrm{ZnO}$ hosts can attract more electrons from the $\mathrm{Zn}-\mathrm{O}$ bonds because of their larger electronegativities, which causes the electron density of the Ga-O bonds to increase and consequently induces shifts in the $\mathrm{Zn} 2 \mathrm{p}$ peaks toward higher binding energies, in contrast to the Ga $2 \mathrm{p}$ peaks, which shift to lower binding energies. ${ }^{36}$

Optical absorption of Ga-doped ZnO NPs. The successful Ga doping of the ZnO NPs was also verified based on their optical characteristics. As shown in Figure 4, the ultraviolet-visible (UV-vis) absorption spectra of the Ga-doped $\mathrm{ZnO}$ NPs were distinctly different from that of the pure $\mathrm{ZnO}$ sample. Clearly, as the doping level increased, the onset of absorption in the doped $\mathrm{ZnO}$ NPs exhibited a gradual blue shift. Their bandgaps were evaluated by linearly extrapolating the $(a h v)^{2}$ vs. photon energy relationships (Figure S1, Supporting Information). The typical computed results are shown in the inset of Figure 4, where bandgaps at 3.48, 3.53, 3.56, 3.61, 3.66 and $3.65 \mathrm{eV}$, corresponding to $\mathrm{Ga}$ dopant contents of $0,1,2$, 4,8 , and 12 at. $\%$, respectively, are plotted; these findings can be attributed to the so-called Moss-Burstein effect. ${ }^{30,44}$ These results are consistent with the scenario in which the Ga dopants introduce a high density of free electrons, which populate the states in the conduction band, ${ }^{30}$ leading to a larger optical bandgap. Note that the bandgap of the $\mathrm{ZnO}$ NPs began to decrease when the Ga doping level exceeded 8 at.\%, which is similar to the behavior observed in previously reported $\mathrm{ZnO}$ NPs at excessively high doping levels. ${ }^{30,47}$ This behavior may indicate the formation of interstitial defects, traps, and even defect clusters above the solubility limit. Based on this observation, the Ga doping levels considered in this work were restricted to the range from 0 to 8 at. $\%$.

Band structures of Ga-doped $\mathrm{ZnO}$ films. Figure 5 shows the ultraviolet photoelectron spectroscopy (UPS) spectra of the valenceband edges and secondary cutoff regions of Ga-doped $\mathrm{ZnO}$ films. The valence-band maximum (VBM) level was estimated from the incident photon energy $(h v, 21.2 \mathrm{eV})$, the onset energy in the valence-band region $\left(\mathrm{E}_{\text {onset }}\right)$ (Figure $5 \mathrm{a}$ ) and the high-binding-energy cutoff $\left(\mathrm{E}_{\text {cutoff }}\right)$ (Figure $5 \mathrm{~b}$ ) using the following equation: $\mathrm{VBM}=21.2-$ $\left(E_{\text {cutoff }}-E_{\text {onset }}\right)$. The approximate VBM positions of doped ZnO NP films with Ga doping levels of 0, 2, and 8 at.\% were found to be $6.98,6.99$ and $7.03 \mathrm{eV}$ below the vacuum level, respectively. This doping dependence is similar to that reported for $\mathrm{Mg}$-doped $\mathrm{ZnO}$ NP films. ${ }^{24}$ Using the bandgaps determined from the UV-vis absorption spectra of the individual NP solid films (Figure S2, Supporting Information), the conduction-band minimum (CBM) levels were estimated to be 3.68, 3.58, and $3.49 \mathrm{eV}$ below the vacuum level for doped $\mathrm{ZnO}$ NP films with Ga doping levels of 0,2 , and 8 at. $\%$, respectively. The approximate VBM and CBM positions of the Ga-doped NPs are summarized in Table 2. Figure $5 \mathrm{c}$ shows the band alignments of Ga-doped $\mathrm{ZnO}$ NPs with different Ga doping levels. After the introduction of the $\mathrm{Ga}$ dopants, the $\mathrm{ZnO}$ bandgap has widened, and the conduction-band edge and valence-band edge have moved in opposite directions. ${ }^{47}$ As we will discuss below, the modified conduction-band edge of a Ga-doped $\mathrm{ZnO}$ NP thin film helps to suppress the charge transfer at the QD/ETL interface, thus suggesting that these low-temperature-processed nanomaterials could be excellent candidates for use as ETLs in highly efficient LED based on CdSe, ${ }^{18,48} \mathrm{CuInS},{ }^{24,49-50}$ and perovskite QDs. ${ }^{51}$
Performance of QD-LEDs with Ga-doped ZnO ETLs. To evaluate the potential of Ga-doped $\mathrm{ZnO}$ NP films for use as the ETLs in QD-LEDs, devices were constructed with a typical configuration of indium tin oxide (ITO)/poly(ethylenedioxythiophene):polystyrene sulfonate (PEDOT:PSS)/poly(9-vinylcarbazole) (PVK)/QDs/Gadoped $\mathrm{ZnO} / \mathrm{Ag}^{12}$ All layers were spin coated onto the patterned ITO substrate except for the Ag cathode, which was deposited via vacuum thermal evaporation. The device structure and the corresponding flat-band energy levels are schematically illustrated in Figures $6 \mathrm{a}$ and $\mathrm{b}$, respectively. The surfaces of the Ga-doped $\mathrm{ZnO}$ films were highly uniform and flat, with a typical root-mean-square roughness below $2 \mathrm{~nm}$ (Figure S3, Supporting Information). Detailed information on the QDs used in the LED devices is provided in Figure S4 (Supporting Information).

Figure $6 \mathrm{c}$ presents the normalized electroluminescence (EL) spectrum of a QD-LED fabricated using $\mathrm{ZnO}$ NPs with a Ga doping level of 8 at.\% as the ETL, and the inset in Figure $6 \mathrm{c}$ presents an emission photograph acquired at a voltage of $5 \mathrm{~V}$, which shows uniform emission across all pixels. The symmetric EL emission peaked at $620 \mathrm{~nm}$ with a FWHM of $36 \mathrm{~nm}$, corresponding to Commission Internationale de l'Eclairage (CIE) color coordinates of $(0.67,0.33)$ (Figure $6 \mathrm{~d})$, which are close to the spectral locus and represent the color-saturated deep red that is ideal for display applications. Compared with the PL spectrum of the QD solution (Figure S5, Table S2, Supporting Information), a slightly red-shifted and broadened EL spectrum was observed, which may be attributable to Förster energy transfer in the close-packed QD solid film and/or an electric-field-induced Stark effect. ${ }^{10,}$ 15, 52 Figure S6 (Supporting Information) shows the EL spectra of the device at various bias voltages, in which no noticeable parasitic emission from the adjacent organic layer (i.e., the PVK) is observed and the shape of the EL spectrum remains unchanged at different biases. These observations confirm that the carriers remain well confined within the $\mathrm{CdSe} / \mathrm{ZnS}$ QD emitting layer and that the recombination zones do not change when the device is subjected to different operating conditions, implying high stability of the as-constructed QD-LED.

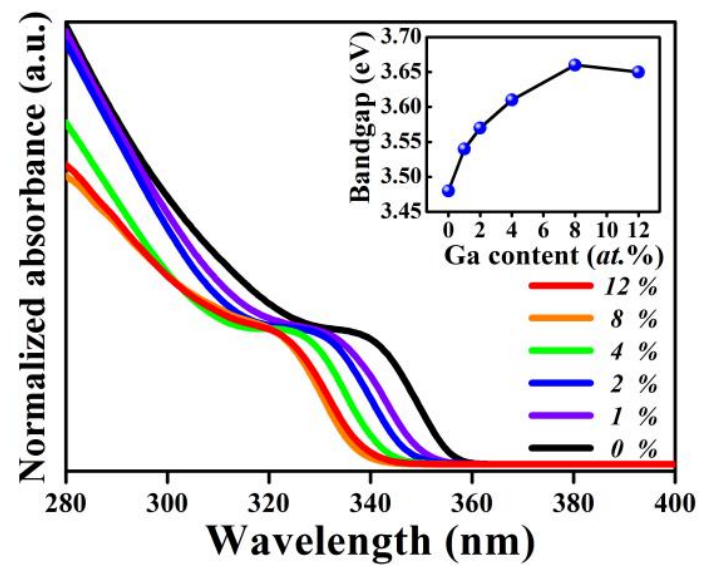

Figure 4. Normalized absorption spectra of Ga-doped ZnO NPs with different nominal Ga doping contents. The inset shows the optical bandgaps of the Ga-doped ZnO NPs. 

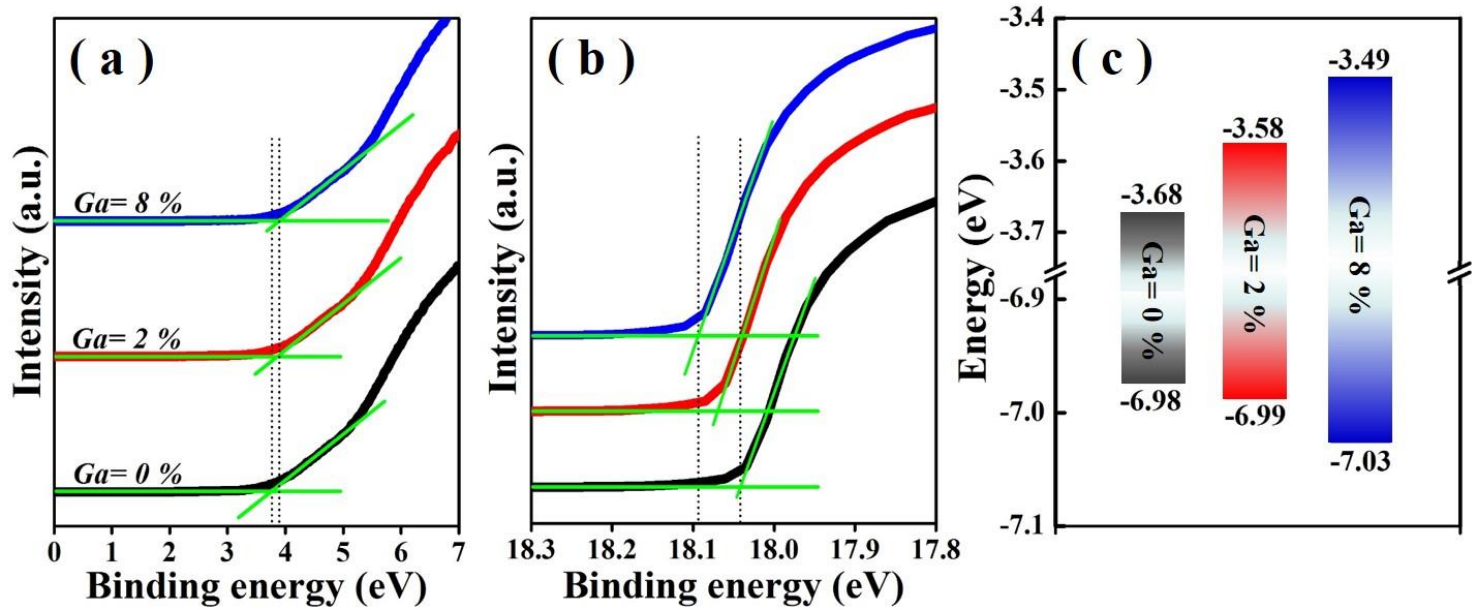

Figure 5. UPS spectra of (a) the valence-band edge regions and (b) the secondary cutoff regions of Ga-doped ZnO NPs from Samples SO (black line), S2 (red line) and S8 (blue line). (c) Band alignments of Ga-doped ZnO NPs with different nominal Ga doping levels.

Table 2. Energy characteristics of Ga-doped ZnO NPs with different nominal Ga doping levels.

\begin{tabular}{lllllll}
\hline $\begin{array}{l}\text { Ga doping level } \\
(\%)\end{array}$ & $\begin{array}{l}\text { Secondary } \\
(\mathrm{eV})\end{array}$ & $\begin{array}{l}\text { cut-off } \\
(\mathrm{eV})\end{array}$ & $\begin{array}{l}\text { Valence band onset } \\
(\mathrm{eV})\end{array}$ & $\begin{array}{l}\text { Valence band maximum }{ }^{a} \\
(\mathrm{eV})\end{array}$ & $\begin{array}{l}\text { Bandgap } \\
(\mathrm{eV})\end{array}$ \\
\hline 0 & 18.03 & 3.19 & 6.98 & 3.30 & 3.68 \\
2 & 18.07 & 3.15 & 6.99 & 3.41 & 3.58 \\
8 & 18.10 & 3.12 & 7.03 & 3.52 & 3.49 \\
\hline
\end{tabular}

: Calculated values according to $V B M=21.2-\left(E_{\text {cutoff }}-E_{\text {onset }}\right)$.

${ }^{b}$ : Calculated values from the optical band gap obtained from UV-vis absorption spectra of doped ZnO film (Figure S3, Supporting Information).

( a )
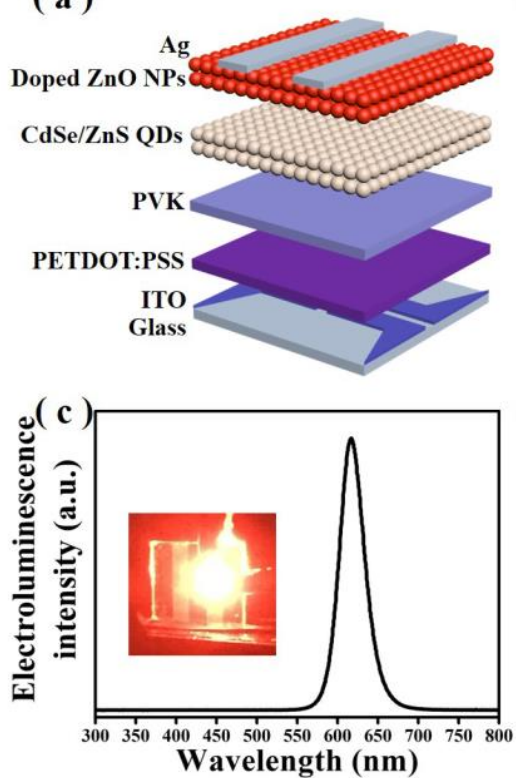
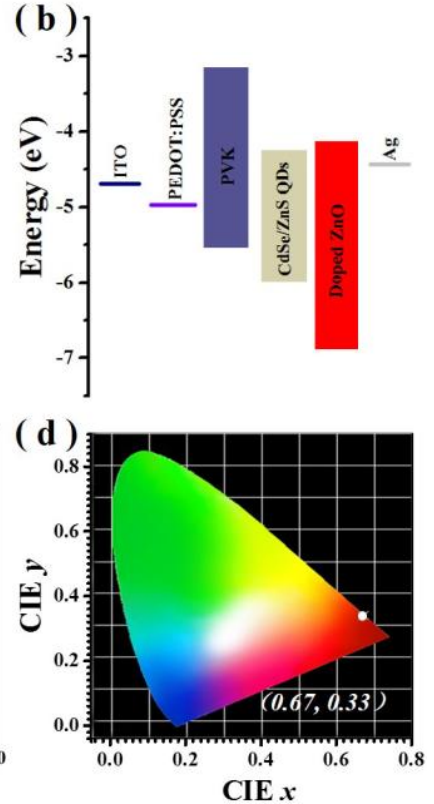

Figure 6. (a) Schematic diagram of the QD-LED configuration. (b) Flat-band energy level diagram of the various layers in a QD-LED. (c) EL spectrum at an applied voltage of $5 \mathrm{~V}$. The inset shows a digital photograph of the QD-LED. (d) Corresponding CIE coordinates. 

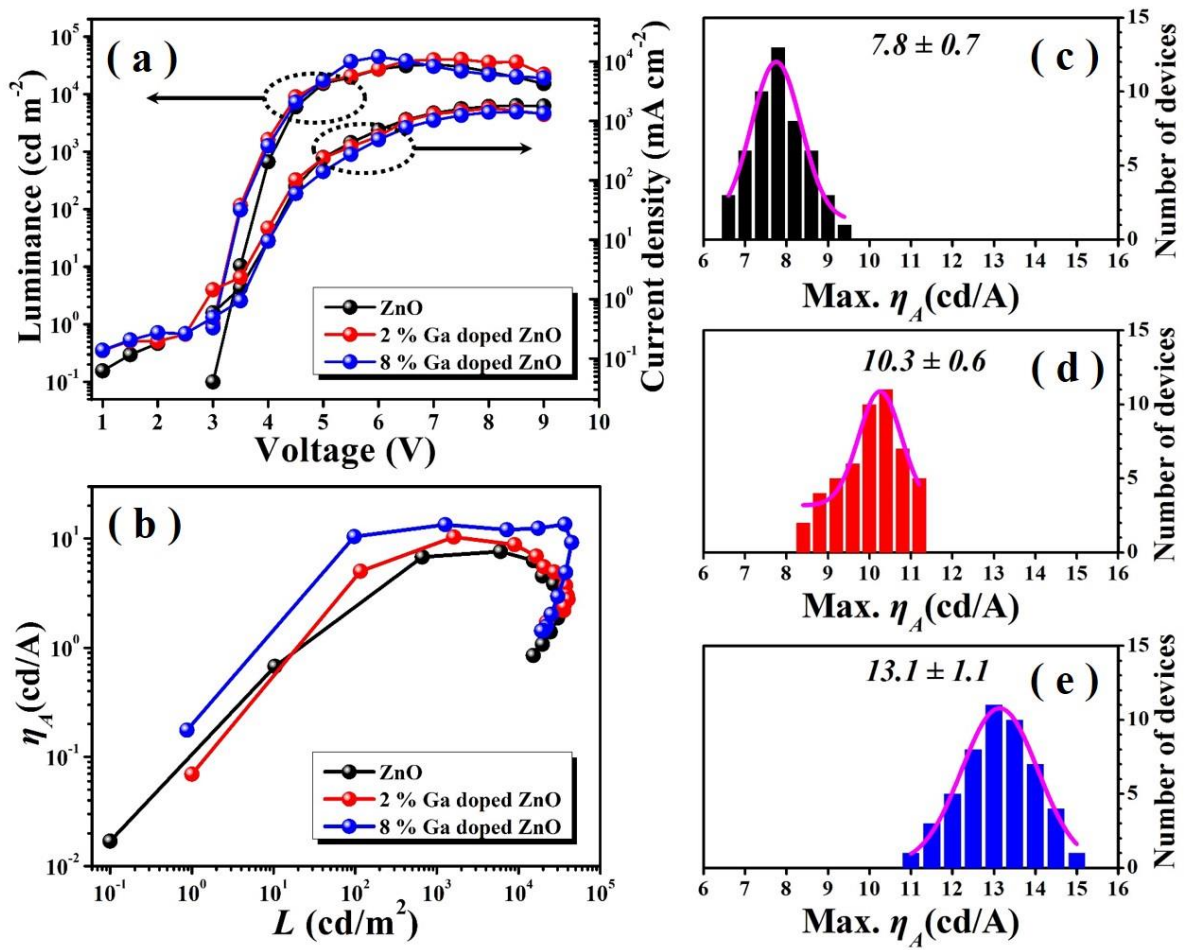

Figure 7. EL performances of QD-LEDs prepared from Samples S0, S2 and S8: (a) Current density (J) and luminance (L) vs. driving voltage (V). (b) Current efficiency $\left(\eta_{\mathrm{A}}\right)$ vs. luminance. (c-d) Statistical distributions of the maximum current efficiencies (max. $\left.\eta_{\mathrm{A}}\right)$ for Samples S0, S2 and S8, each evaluated for 50 devices.

To investigate the effect of the $\mathrm{Ga}$ doping content of the $\mathrm{ZnO}$ NPs on QD-LED performance, ZnO NPs from Samples S0, S2 and S8 were utilized as the ETL layers for device construction. The resulting EL performances are shown in Figure 7. The variations in current density and luminance with applied voltage are presented in Figure 7a. The turn-on voltages of the QD-LEDs are centered at $\sim 3$ $\mathrm{V}$, and their corresponding brightnesses are $0.1,0.9$ and 0.87 $\mathrm{cd} / \mathrm{m}^{2}$ for Samples S0, S2 and S8, respectively. The maximum brightnesses are 33000 (@7 V), 40000 (@7 V), and 44000 $\mathrm{cd} / \mathrm{m}^{2}$ (@6.5 V), respectively, suggesting that the introduction of Ga dopants into ZnO ETLs can result in significant performance enhancement for QD-LEDs. The characteristic current efficiency curves as a function of luminance are plotted in Figure $7 \mathrm{~b}$, and Figures $7 \mathrm{c}$-e present the statistical distributions of $\eta_{\mathrm{A}}$ collected from 50 devices for each of the three device types. The mean $\eta_{\mathrm{A}}$ values for Samples SO, S2 and S8 are 7.8, 10.3 and $13.1 \mathrm{~cd} / \mathrm{A}$ (with a maximum observed value of $15 \mathrm{~cd} / \mathrm{A}$ ), respectively, which are comparable to the highest efficiency ever reported for red-light QD-LEDs (Table S3, Supporting Information). Furthermore, the efficiency of a QD-LED with 8 at.\% Ga doping can be enhanced by more than 1.5 times compared with its undoped counterpart (an increase of up to $168 \%$, i.e., from 7.8 to $13.1 \mathrm{~cd} / \mathrm{A}$ ), thereby confirming that Ga-doped ZnO NP ETLs enable the fabrication of QD-LEDs with significantly improved performance.

The QD-LED performance improvement enabled by a Ga-doped $\mathrm{ZnO}$ NP ETL can be attributed to weakened exciton dissociation in the emitters at the QD/ETL interface. Figure 8a shows the timeresolved PL dynamics of QD films on ITO glass substrates with and without $\mathrm{ZnO}$ layers. The lifetime of the QDs is obviously shortened in the presence of a pure $\mathrm{ZnO}$ film, indicating considerable exciton dissociation in the QD emitters at the $\mathrm{QD} / \mathrm{ZnO}$ interface. When the pure $\mathrm{ZnO}$ layer is replaced with a Ga-doped $\mathrm{ZnO}$ film, the decay becomes slower as the Ga doping level increases, thereby confirming that the introduction of Ga dopants into the $\mathrm{ZnO}$ NP ETL is an effective means of weakening the exciton dissociation in a QD-LED device. To further elucidate the charge transfer behavior, the data were fitted with a single-exponential function of the form $(t)=A \exp (-\mathrm{t} / \tau){ }^{2}{ }^{2}{ }^{14}$ The PL decay lifetime of CdSe/ZnS QDs on glass was found to be $13.45 \mathrm{~ns}$, whereas those for the QD films that were overcoated with doped $\mathrm{ZnO}$ layers were 9.61, 11.09 and $11.93 \mathrm{~ns}$ for $\mathrm{Ga}$ doping levels of 0,2 , and 8 at. $\%$, respectively. The charge transfer rate $k_{\mathrm{ET}}$ and efficiency $\eta_{\mathrm{ET}}$ can be calculated by: ${ }^{19,53} k_{\mathrm{ET}}=$ $1 / \tau_{\mathrm{QD} / \text { doped }^{-} \mathrm{ZnO}^{-}}-1 / \tau_{\mathrm{QD}}$ and $\eta_{E T}=1-\tau_{\mathrm{QD} / \text { doped }^{-} \mathrm{ZnO}} / \tau_{\mathrm{QD}}$, respectively, which are summarized in Table 3. The spontaneous charge transfer rates and efficiencies both gradually decrease as the Ga doping level increases, which is consistent with Marcus theory, ${ }^{54}$ suggesting that the charge transfer rate between the QDs and the adjacent NPs is dominated by the energy offset $(\Delta \mathrm{G})$ between their conduction-band levels. In our case, the $\Delta \mathrm{G}$ between the QDs and the adjacent Gadoped $\mathrm{ZnO}$ NPs is reduced because of the increase in the conduction-band level of the $\mathrm{ZnO}$ NPs caused by the Ga dopants. ${ }^{18,21,54}$ The exciton dissociation in the QDs that is induced by charge transfer to the adjacent ETL will cause the PL QY of the QDs to decrease, leading to low efficiency of the QD-LED. These results verify that the Ga doping of the $\mathrm{ZnO}$ NP ETL helps to maintain the charge neutrality of the QD emitters and to preserve their superior emissive properties.

To reduce nonradiative recombination and increase device efficiency, it is critical to engineer the charge transport layers to achieve balanced charge transport and low leakage currents. ${ }^{2,18}$ Hence, 
strategies such as tailoring the ETL and/or introducing an insulating layer between the ETL and the emissive layer have been explored for optimizing device performance. ${ }^{2}$ In our case, to reveal the charge transport characteristics of ZnO NP ETLs with different Ga doping levels, the current density-voltage $(J-V)$ characteristics of electron-only devices based on Ga-doped $\mathrm{ZnO}$ NPs were investigated, ${ }^{9,55}$ as presented in Figure 8b. Clearly, as the Ga doping level increases, the junction current is reduced, thereby confirming that the incorporation of Ga dopants into $\mathrm{ZnO}$ NP ETLs is an effective means of reducing nonradiative charge recombination ${ }^{43}$ and, consequently, boosting the efficiency of QD-LED devices.
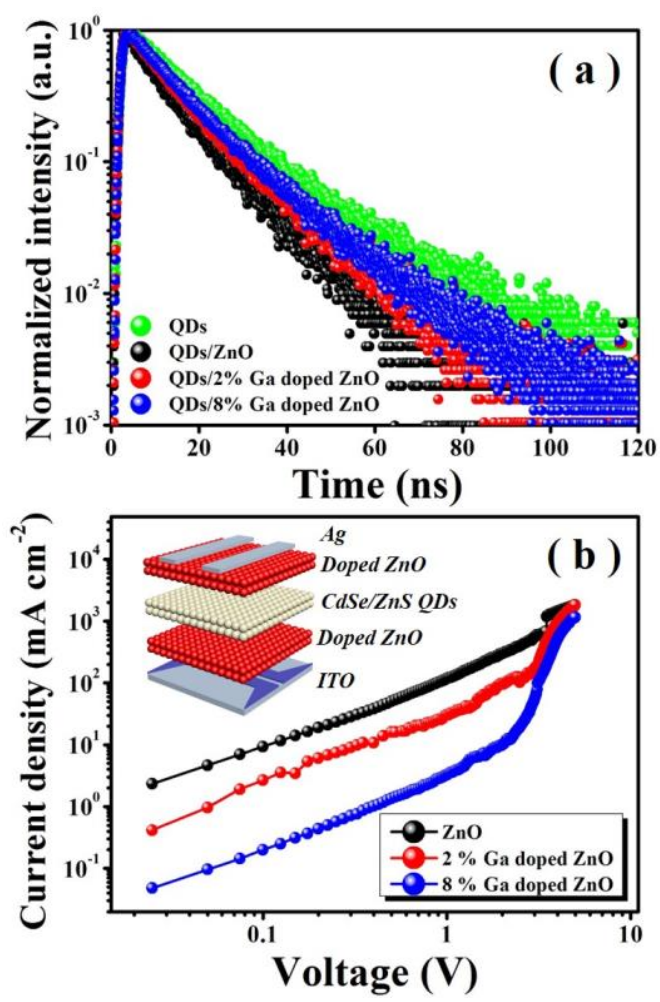

Figure 8. (a) Time-resolved PL dynamics of QD films without a $\mathrm{ZnO}$ layer and with $\mathrm{ZnO}$ NP layers prepared from Samples S0, S2 and S8 on ITO substrates. (b) Current density-voltage $(J-V)$ characteristics of electron-only devices based on Ga-doped ZnO NPs. The insert shows a schematic illustration of an as-constructed electron-only QD-LED, with a configuration of ITO/doped $\mathrm{ZnO} / \mathrm{CdSe} / \mathrm{ZnS}$ QDs/doped $\mathrm{ZnO} / \mathrm{Ag}$.

Table 3. The rate $\left(k_{E T}\right)$ and efficiency $\left(\eta_{E T}\right)$ of spontaneous charge transfer from CdSe/ZnS QDs to Ga-doped ZnO ETLs.

\begin{tabular}{lll}
\hline Films & $k_{\mathrm{ET}}\left(10^{7} \mathrm{~S}^{-1}\right)$ & $\eta_{\mathrm{ET}}(\%)$ \\
\hline QDs/ pure ZnO & 2.97 & 28.6 \\
QDs/ZnO with 2\% Ga ions & 1.58 & 17.5 \\
QDs/ZnO with 8\% Ga ions & 0.95 & 11.3 \\
\hline
\end{tabular}

\section{CONCLUSIONS}

In conclusion, we reported the exploration of highly bright and efficient QD-LEDs with ETLs consisting of Ga-doped ZnO NPs, which were grown via a RT solution process without the need for any post-treatment or bulky organic ligands. By varying the nominal
Ga content from 0 to 12 at.\%, high-quality and monodisperse Gadoped $\mathrm{ZnO}$ NPs with good crystallinity were obtained. The Ga dopants lowered the work functions and tailored the band structures of their $\mathrm{ZnO}$ hosts. The as-assembled QD-LEDs with ETLs consisting of Ga-doped $\mathrm{ZnO}$ NPs exhibited superior luminances of $\sim 33000,40000$, and $44000 \mathrm{~cd} / \mathrm{m}^{2}$ and mean current efficiencies of $7.8,10.3$, and $13.1 \mathrm{~cd} / \mathrm{A}$ (with a maximum efficiency of $15 \mathrm{~cd} / \mathrm{A}$ ) for Ga doping levels of 0, 2 and 8 at.\%, respectively; these efficiencies are comparable to the highest value ever reported for red-light QD-LEDs. The significantly enhanced brightness and efficiency of these QD-LEDs are attributed to the Ga dopants, which facilitate the transfer of electrons to the adjacent QD layer and limit the quenching of the exciton emission in the QDs. Our work thus introduces low-temperature-processed Ga-doped ZnO NPs that enable the fabrication of high-performance QD-LEDs for a wide range of optoelectronics applications.

\section{EXPERIMENTAL METHODS}

Materials. Commercially available zinc acetate $\left(\mathrm{Zn}(\mathrm{Ac})_{2}\right)$, dimethyl sulfoxide (DMSO), and tetramethylammonium hydroxide pentahydrate (TMAH) were obtained from Adamas-beta, China. Gallium triacetate $\left(\mathrm{Ga}(\mathrm{Ac})_{3}\right)$ was purchased from Sigma-Aldrich, USA. PEDOT:PSS and PVK were purchased from Xi'an Polymer Light Technology Corp., China. Cadmium selenide/zinc sulfide alloyed (CdSe/ZnS) QDs with a typical PL QY of $75 \%$ were purchased from Najing Technology Corp., China.

Synthesis of $\mathrm{ZnO}$ NPs. The $\mathrm{ZnO}$ NPs were prepared via the hydrolysis of $\mathrm{Zn}$ salts in basic solutions. ${ }^{56}$ In a typical experiment, a stoichiometric amount of TMAH dissolved in ethanol $(0.5 \mathrm{M})$ was dropwise added to $0.1 \mathrm{M}$ zinc acetate dihydrate $\left(\mathrm{Zn}(\mathrm{Ac})_{2} \cdot 2 \mathrm{H}_{2} \mathrm{O}\right)$ dissolved in DMSO, and the reaction was allowed to proceed at 30 ${ }^{\circ} \mathrm{C}$ for $1 \mathrm{~h}$ to synthesize $\mathrm{ZnO}$ NPs. The as-synthesized NPs were precipitated with excess acetone and then completely redispersed in ethanol for optical characterization and ETL spin deposition. Using a similar method, Ga-doped ZnO NPs with nominal doping levels ranging from 0 to 12 at. $\%$ were produced by varying the initial ratio of the gallium acetate precursor with respect to the zinc acetate while keeping the other parameters constant. The obtained NPs with nominal doping levels of 0, 1, 2, 4, 8 and 12 at.\% were labeled as Samples S0, S1, S2, S4, S8 and S12, respectively.

Fabrication and Characterization of QD-LEDs. QD-LEDs were fabricated on glass substrates coated with ITO. The substrates were consecutively cleaned with deionized acetone, ethanol, and water for $15 \mathrm{~min}$ each and were then treated for $10 \mathrm{~min}$ with ozone generated by ultraviolet light in air. Then, the substrates were spin coated with multiple layers in the following order: PEDOT:PSS, PVK, CdSe/ZnS QDs, and doped ZnO NPs. First, the PEDOT:PSS solution was spin coated onto the ITO-coated glass substrates at $3500 \mathrm{rpm}$ for $45 \mathrm{~s}$, followed by baking at $150^{\circ} \mathrm{C}$ for $15 \mathrm{~min}$. Then, the coated substrates were transferred into an $\mathrm{N}_{2}$-filled glove box for the spin coating of the PVK, QD and $\mathrm{ZnO}$ layers. The PVK (10 $\mathrm{mg} / \mathrm{mL}$, chlorobenzene) was deposited at a fixed speed of $2000 \mathrm{rpm}$ for $30 \mathrm{~s}$, followed by baking at $100{ }^{\circ} \mathrm{C}$ for $10 \mathrm{~min}$. The CdSe/ZnS QDs were dissolved in toluene $(15 \mathrm{mg} / \mathrm{mL})$ and then spin coated at $2000 \mathrm{rpm}$ for $30 \mathrm{~s}$, followed by annealing at $100{ }^{\circ} \mathrm{C}$ for $10 \mathrm{~min}$. Subsequently, the doped $\mathrm{ZnO}$ NP layers were deposited at a speed of $2000 \mathrm{rpm}$ for $30 \mathrm{~s}$ from a $30 \mathrm{mg} / \mathrm{mL}$ ethanol solution. Afterward, the multilayer samples were loaded into a custom highvacuum deposition chamber (background pressure: $\sim 1 \times 10^{-3} \mathrm{~Pa}$ ) for 
the deposition of the top Ag cathode (thickness: $~ 100 \mathrm{~nm}$ ), which was patterned using an in situ shadow mask to form an active device area of $5 \mathrm{~mm}^{2}$

Structure Characterization and Optical Property Measurements. The obtained NPs were characterized in terms of morphology, microstructure, and composition using HRTEM (JEM-2100F, JEOL, Japan), XRD (D8 Advance, Bruker, Germany), inductively coupled plasma optical emission spectrometry (ICP-OES; Ultima 2, Horiba Jobin Yvon, France) and XPS (Thermo ESCALAB 250XI, America). For determining the chemical composition by ICP-OES, the doped $\mathrm{ZnO}$ NP samples were prepared by dissolving the purified oxide nanocrystals in concentrated nitric acid. UV-vis measurements of the obtained NPs were performed using a UV-vis scanning spectrophotometer (U-3900, Hitachi, Japan). The electronic structures and surface morphologies of the doped $\mathrm{ZnO} \mathrm{NP}$ films were investigated via UPS (AXIS Ultra DLD, Shimadzu/Kratos Inc., Japan) and atomic force microscopy (AFM; Nanoscope V, Veeco, USA), respectively. For the UPS measurements, Ga-doped $\mathrm{ZnO}$ NP solid films were prepared by spin casting the different NP dispersions onto ITO glass substrates. PL spectra, PL QYs, and PL decay curves were recorded using a spectrometer (Fluromax-4P, Horiba Jobin Yvon, France) equipped with a quantum-yield accessory and a time-correlated single-photon-counting (TCSPC) spectrometer. A NanoLED pulsed light source with a wavelength of 450 $\mathrm{nm}$ was utilized as the exciting source for the PL decay measurements. The PL decay curves were analyzed using the DAS6 software package.

\section{ASSOCIATED CONTENT}

\section{Supporting Information}

Additional data include the calculated lattice parameters (Table S1) and bandgaps (Figure S1) of ZnO NPs, the bandgaps (Figure S2) and surface morphology (Figure S3) of ZnO NPs films, the optical and structural characteristics of CdSe/ZnS QDs (Figure S4), and the EL result of QD-LEDs (Figure S5-6, Table S2-3). This material is available free of charge via the Internet at http://pubs.acs.org

\section{AUTHOR INFORMATION}

\section{Corresponding Author}

E-mail: smh2875@163.com (M. Shang)

$$
\text { tao.wu@kaust.edu.sa }(T . W u)
$$

\section{Author Contributions}

${ }^{\square}$ S. C. and J. J. Z. contributed equally.

\section{Notes}

The authors declare no competing financial interests.

\section{ACKNOWLEDGMENT}

This work was financially supported by National Natural Science Foundation of China (NSFC, Grant No. 61106066), Zhejiang Provincial Science Foundation (Grant No. LY14F040001), Foundation of Educational Commission in Zhejiang Province of China (Grant No. Y201533502) and Natural Science Foundation of Ningbo Municipal Government (Grant Nos. 2016A610104 and 2016A610108).

\section{REFERENCES}

(1) Colvin, V. L.; Schlamp, M. C.; Alivisatos, A. P. LightEmitting Diodes Made from Cadmium Selenide Nanocrystals and a Semiconducting Polymer. Nature 1994, 370, 354-357.

(2) Dai, X.; Zhang, Z.; Jin, Y.; Niu, Y.; Cao, H.; Liang, X.; Chen, L.; Wang, J.; Peng, X. Solution-Processed, High-Performance Light-Emitting Diodes Based on Quantum Dots. Nature 2014, 515, 96-99.

(3) Anikeeva, P. O.; Halpert, J. E.; Bawendi, M. G.; Bulović, V. Quantum Dot Light-Emitting Devices with Electroluminescence Tunable over the Entire Visible Spectrum. Nano Lett. 2009, 9, 2532-2536.

(4) Caruge, J.-M.; Halpert, J. E.; Bulović, V.; Bawendi, M. G. $\mathrm{NiO}$ as an Inorganic Hole-Transporting Layer in QuantumDot Light-Emitting Devices. Nano Lett. 2006, 6, 2991-2994.

(5) Cho, K.-S.; Lee, E. K.; Joo, W.-J.; Jang, E.; Kim, T.-H.; Lee, S. J.; Kwon, S.J.; Han, J. Y.; Kim, B.-K.; Choi, B. L.; Kim, J. M. High-Performance Crosslinked Colloidal Quantum-Dot Light-Emitting Diodes. Nat. Photonics 2009, 3, 341-345.

(6) Shirasaki, Y.; Supran, G. J.; Bawendi, M. G.; Bulovic, V. Emergence of Colloidal Quantum-Dot Light-Emitting Technologies. Nat. Photonics 2013, 7 (1), 13-23.

(7) Yang, X.; Mutlugun, E.; Dang, C.; Dev, K.; Gao, Y.; Tan, S. T.; Sun, X. W.; Demir, H. V. Highly Flexible, Electrically Driven, Top-Emitting, Quantum Dot Light-Emitting Stickers. ACS Nano 2014, 8, 8224-8231.

(8) Mashford, B. S.; Stevenson, M.; Popovic, Z.; Hamilton, C.; Zhou, Z.; Breen, C.; Steckel, J.; Bulovic, V.; Bawendi, M.; Coe-Sullivan, S.; Kazlas, P. T. High-Efficiency Quantum-Dot Light-Emitting Devices with Enhanced Charge Injection. Nat. Photonics 2013, 7, 407-412.

(9) Shen, H.; Cao, W.; Shewmon, N. T.; Yang, C.; Li, L. S.; Xue, J. High-Efficiency, Low Turn-on Voltage Blue-Violet Quantum-Dot-Based Light-Emitting Diodes. Nano Lett. 2015, 15, 1211-1216.

(10) Zhao, J.; Bardecker, J. A.; Munro, A. M.; Liu, M. S.; Niu, Y.; Ding, I. K.; Luo, J.; Chen, B.; Jen, A. K. Y.; Ginger, D. S. Efficient CdSe/CdS Quantum Dot Light-Emitting Diodes Using a Thermally Polymerized Hole Transport Layer. Nano Lett. 2006, 6, 463-467.

(11) Qian, L.; Zheng, Y.; Xue, J.; Holloway, P. H. Stable and Efficient Quantum-Dot Light-Emitting Diodes Based on Solution-Processed Multilayer Structures. Nat. Photonics 2011, 5, 543-548.

(12) Lee, K.-H.; Lee, J.-H.; Kang, H.-D.; Park, B.; Kwon, Y.; Ko, H.; Lee, C.; Lee, J.; Yang, H. Over 40 cd/A Efficient Green Quantum Dot Electroluminescent Device Comprising Uniquely Large-Sized Quantum Dots. ACS Nano 2014, 8, 4893-4901.

(13) Shen, H.; Bai, X.; Wang, A.; Wang, H.; Qian, L.; Yang, Y.; Titov, A.; Hyvonen, J.; Zheng, Y.; Li, L. S. High-Efficient Deep-Blue Light-Emitting Diodes by Using High Quality $\mathrm{Zn}_{\mathrm{x}} \mathrm{Cd}_{1-\mathrm{x}} \mathrm{S} / \mathrm{ZnS}$ Core/Shell Quantum Dots. Adv. Funct. Mater. 2014, 24, 2367-2373.

(14) Gao, Y.; Peng, X. Photogenerated Excitons in Plain Core CdSe Nanocrystals with Unity Radiative Decay in Single Channel: The Effects of Surface and Ligands. J. Am. Chem. 
Soc. 2015, 137, 4230-4235.

(15) Shirasaki, Y.; Supran, G. J.; Tisdale, W. A.; Bulović, V. Origin of Efficiency Roll-Off in Colloidal Quantum-Dot LightEmitting Diodes. Phys. Rev. Lett. 2013, 110, 217403.

(16) Ji, W.; Jing, P.; Zhang, L.; Li, D.; Zeng, Q.; Qu, S.; Zhao, J. The Work Mechanism and Sub-Bandgap-Voltage Electroluminescence in Inverted Quantum Dot LightEmitting Diodes. Sci. Rep. 2014, 4, 6974.

(17) Dong, Y.; Caruge, J.-M.; Zhou, Z.; Hamilton, C.; Popovic, Z.; Ho, J.; Stevenson, M.; Liu, G.; Bulovic, V.; Bawendi, M.; Kazlas, P. T.; Steckel, J.; Coe-Sullivan, S. 20.2: Ultra-Bright, Highly Efficient, Low Roll-Off Inverted Quantum-Dot Light Emitting Devices (QLEDs). Dig. Tech. Pap. - Soc. Inf. Disp. 2015, 46, 270-273.

(18) Pan, J.; Chen, J.; Huang, Q.; Khan, Q.; Liu, X.; Tao, Z.; Zhang, Z.; Lei, W.; Nathan, A. Size Tunable ZnO Nanoparticles To Enhance Electron Injection in Solution Processed QLEDs. ACS Photonics 2016, 3, 215-222.

(19) Kongkanand, A.; Tvrdy, K.; Takechi, K.; Kuno, M.; Kamat, P. V. Quantum Dot Solar Cells. Tuning Photoresponse through Size and Shape Control of $\mathrm{CdSe}-\mathrm{TiO}_{2}$ Architecture. J. Am. Chem. Soc. 2008, 130, 4007-4015.

(20) Wu, X.; Yeow, E. K. L. Charge-Transfer Processes in Single $\mathrm{CdSe} / \mathrm{ZnS}$ Quantum Dots with p-Type NiO Nanoparticles. Chem. Commum. 2010, 46, 4390-4392.

(21) Jin, S.; Lian, T. Electron Transfer Dynamics from Single CdSe/ZnS Quantum Dots to $\mathrm{TiO}_{2}$ Nanoparticles. Nano Lett. 2009, 9, 2448-2454.

(22) Zhou, Y.; Fuentes-Hernandez, C.; Shim, J.; Meyer, J.; Giordano, A. J.; Li, H.; Winget, P.; Papadopoulos, T.; Cheun, H.; Kim, J.; Fenoll, M.; Dindar, A.; Haske, W.; Najafabadi, E.; Khan, T. M.; Sojoudi, H.; Barlow, S.; Graham, S.; Brédas, J.-L.; Marder, S. R.; Kahn, A.; Kippelen, B. A Universal Method to Produce Low-Work Function Electrodes for Organic Electronics. Science 2012, 336, 327-332.

(23) Kim, H. H.; Park, S.; Yi, Y.; Son, D. I.; Park, C.; Hwang, D. K.; Choi, W. K. Inverted Quantum Dot Light Emitting Diodes using Polyethylenimine Ethoxylated Modified $\mathrm{ZnO}$. Sci. Rep. 2015, 5, 8968.

(24) Kim, J.-H.; Han, C.-Y.; Lee, K.-H.; An, K.-S.; Song, W.; Kim, J.; Oh, M. S.; Do, Y. R.; Yang, H. Performance Improvement of Quantum Dot-Light-Emitting Diodes Enabled by an Alloyed $\mathrm{ZnMgO}$ Nanoparticle Electron Transport Layer. Chem. Mater. 2015, 27, 197-204.

(25) Li, X.; Liu, Y.; Song, J.; Xu, J.; Zeng, H. MgZnO Nanocrystals: Mechanism for Dopant-Stimulated Self-Assembly. Small 2015, 11, 5097-5104.

(26) Hewlett, R. M.; McLachlan, M. A. Surface Structure Modification of $\mathrm{ZnO}$ and the Impact on Electronic Properties. Adv. Mater. 2016, 28, 3893-3921.

(27) Prosa, M.; Tessarolo, M.; Bolognesi, M.; Margeat, O.; Gedefaw, D.; Gaceur, M.; Videlot-Ackermann, C.; Andersson, M. R.; Muccini, M.; Seri, M.; Ackermann, J. Enhanced Ultraviolet Stability of Air-Processed Polymer Solar Cells by Al Doping of the $\mathrm{ZnO}$ Interlayer. ACS Appl. Mater. Interfaces 2016, 8, 1635-1643.

(28) Song, J.; Kulinich, S. A.; Li, J.; Liu, Y.; Zeng, H. A General One-Pot Strategy for the Synthesis of High-Performance Transparent-Conducting-Oxide Nanocrystal Inks for All-
Solution-Processed Devices. Angew. Chem., Int. Ed. 2015, 127, $472-476$.

(29) Song, J.; Zeng, H. Transparent Electrodes Printed with Nanocrystal Inks for Flexible Smart Devices. Angew. Chem., Int. Ed. 2015, 54, 9760-9774.

(30) Liang, X.; Ren, Y.; Bai, S.; Zhang, N.; Dai, X.; Wang, X.; He, H.; Jin, C.; Ye, Z.; Chen, Q.; Chen, L.; Wang, J.; Jin, Y. Colloidal Indium-Doped Zinc Oxide Nanocrystals with Tunable Work Function: Rational Synthesis and Optoelectronic Applications. Chem. Mater. 2014, 26, 5169. 5178.

(31) Della Gaspera, E.; Bersani, M.; Cittadini, M.; Guglielmi, M.; Pagani, D.; Noriega, R.; Mehra, S.; Salleo, A.; Martucci, A. Low-Temperature Processed Ga-Doped $\mathrm{ZnO}$ Coatings from Colloidal Inks. J. Am. Chem. Soc. 2013, 135, 3439-3448.

(32) Buonsanti, R.; Llordes, A.; Aloni, S.; Helms, B. A.; Milliron, D. J. Tunable Infrared Absorption and Visible Transparency of Colloidal Aluminum-Doped Zinc Oxide Nanocrystals. Nano Lett. 2011, 11, 4706-4710.

(33) Hamza, M. K.; Bluet, J. M.; Masenelli-Varlot, K.; Canut, B.; Boisron, O.; Melinon, P.; Masenelli, B. Tunable Mid IR Plasmon in GZO Nanocrystals. Nanoscale 2015, 7, 12030 12037.

(34) Della Gaspera, E.; Chesman, A. S. R.; van Embden, J.; Jasieniak, J. J. Non-injection Synthesis of Doped Zinc Oxide Plasmonic Nanocrystals. ACS Nano 2014, 8, 9154-9163.

(35) Lee, S. Y.; Song, Y.W.; Jeon, K. A. Synthesis and Analysis of Resistance-Controlled Ga-doped $\mathrm{ZnO}$ Nanowires. J. Cryst. Growth 2008, 310, 4477-4480.

(36) Zhang, X.; Li, L.; Su, J.; Wang, Y.; Shi, Y.; Ren, X.; Liu, N.; Zhang, A.; Zhou, J.; Gao, Y. Bandgap Engineering of $\mathrm{Ga}_{\mathrm{x}} \mathrm{Zn}_{1-}$ ${ }_{\mathrm{x}} \mathrm{O}$ Nanowire Arrays for Wavelength-Tunable Light-Emitting Diodes. Laser Photonics Rev. 2014, 8, 429-435.

(37) Park, G. C.; Hwang, S. M.; Lim, J. H.; Joo, J. Growth Behavior and Electrical Performance of Ga-doped $\mathrm{ZnO}$ Nanorod/p-Si Heterojunction Diodes Prepared Using a Hydrothermal Method. Nanoscale 2014, 6, 1840-1847.

(38) Haifeng, Z.; Hua, W.; Xingyou, T.; Kang, Z.; Fei, X.; Zheng, S.; Konghu, T.; Qiulong, 1.; Fei, F. Solvothermal Synthesis of Gallium-Doped Zinc Oxide Nanoparticles with Tunable Infrared Absorption. Mater. Res. Express 2014, 1, 045022.

(39) Saha, M.; Ghosh, S.; Ashok, V. D.; De, S. K. Carrier Concentration Dependent Optical and Electrical Properties of Ga Doped $\mathrm{ZnO}$ Hexagonal Nanocrystals. Phys. Chem. Chem. Phys. 2015, 17, 16067-16079.

(40) Boles, M. A.; Ling, D.; Hyeon, T.; Talapin, D. V. The Surface Science of Nanocrystals. Nat. Mater. 2016, 15 (2), 141-153.

(41) Zhao, B.; Wang, F.; Chen, H.; Wang, Y.; Jiang, M.; Fang, X.; Zhao, D. Solar-Blind Avalanche Photodetector Based On Single $\mathrm{ZnO}-\mathrm{Ga}_{2} \mathrm{O}_{3}$ Core-Shell Microwire. Nano Lett. 2015, 15, 3988-3993.

(42) Thambidurai, M.; Kim, J. Y.; Song, J.; Ko, Y.; Song, H.-j.; Kang, C.-m.; Muthukumarasamy, N.; Velauthapillai, D.; Lee, C. High Performance Inverted Organic Solar Cells with Solution Processed Ga-doped $\mathrm{ZnO}$ as an Interfacial Electron Transport Layer. J. Mater. Chem. C 2013, 1, 8161-8166.

(43) Wu, H.-C.; Peng, Y.-C.; Chen, C.-C. Effects of Ga Concentration on Electronic and Optical Properties of Gadoped $\mathrm{ZnO}$ from First Principles Calculations. Opt. Mater. 
2013, 35, 509-515.

(44) Zeng, W.; Yang, X.; Shang, M.; Xu, X.; Yang, W.; Hou, H. Fabrication of Mg-doped ZnO Nanofibers with High Purities and Tailored Band Gaps. Ceram. Int. 2016, 42, 10021-10029.

(45) Wang, X.; Jin, Y.; He, H.; Yang, F.; Yang, Y.; Ye, Z. Bandgap Engineering and Shape Control of Colloidal $\mathrm{Cd}_{\mathrm{x}} \mathrm{Zn}_{1-\mathrm{x}} \mathrm{O}$ Nanocrystals. Nanoscale 2013, 5, 6464-6468.

(46) Yuan, G. D.; Zhang, W. J.; Jie, J. S.; Fan, X.; Tang, J. X.; Shafiq, I.; Ye, Z. Z.; Lee, C. S.; Lee, S. T. Tunable n-Type Conductivity and Transport Properties of Ga-doped $\mathrm{ZnO}$ Nanowire Arrays. Adv. Mater. 2008, 20, 168-173.

(47) Cohn, A. W.; Kittilstved, K. R.; Gamelin, D. R. Tuning the Potentials of "Extra" Electrons in Colloidal n-Type $\mathrm{ZnO}$ Nanocrystals via $\mathrm{Mg}^{2+}$ Substitution. J. Am. Chem. Soc.2012, 134, 7937-7943.

(48) Zhang, H.; Sun, X.; Chen, S. Over $100 \mathrm{~cd} \mathrm{~A}$ A $^{-1}$ Efficient Quantum Dot Light-Emitting Diodes with Inverted Tandem Structure. Adv. Funct. Mater. 2017, DOI: 10.1002/adfm.201700610.

(49) Bai, Z.; Ji, W.; Han, D.; Chen, L.; Chen, B.; Shen, H.; Zou, B.; Zhong, H. Hydroxyl-Terminated $\mathrm{CuInS}_{2}$ Based Quantum Dots: Toward Efficient and Bright Light Emitting Diodes. Chem. Mater. 2016, 28, 1085-1091.

(50) Chang, S.; Zhang, X.; Wang, Z.; Han, D.; Tang, J.; Bai, Z.; Zhong, H. Alcohol-Soluble Quantum Dots: Enhanced Solution Processability and Charge Injection for Electroluminescence Devices. IEEE J. Sel. Top. Quantum Electron. 2017, DOI: 10.1109/JSTQE.2017.2688706.

(51) Qasim, K.; Wang, B.; Zhang, Y.; Li, P.; Wang, Y.; Li, S.; Lee, S.-T.; Liao, L.-S.; Lei, W.; Bao, Q. Solution-Processed Extremely Efficient Multicolor Perovskite Light-Emitting Diodes Utilizing Doped Electron Transport Layer. Adv. Funct. Mater. 2017, DOI: 10.1002/adfm.201606874.

(52) Cao, S.; Ji, W.; Zhao, J.; Yang, W.; Li, C.; Zheng, J. ColorTunable Photoluminescence of Cu-doped Zn-In-Se Quantum Dots and Their Electroluminescence Properties. J. Mater. Chem. C 2016, 4 , 581-588.

(53) Sun, M.; Zhu, D.; Ji, W.; Jing, P.; Wang, X.; Xiang, W.; Zhao, J. Exploring the Effect of Band Alignment and Surface States on Photoinduced Electron Transfer from $\mathrm{CuInS}_{2} / \mathrm{CdS}$ Core/Shell Quantum Dots to $\mathrm{TiO}_{2}$ Electrodes. ACS Appl. Mater. Interfaces 2013, 5, 12681-12688.

(54) Marcus, R. A. On the Theory of Oxidation-Reduction Reactions Involving Electron Transfer. I. J. Chem. Phys. 1956, 24, 966-978.

(55) Lin, Q.; Chen, F.; Wang, H.; Shen, H.; Wang, A.; Wang, L.; Zhang, F.; Guo, F.; Li, L. S. Influence of Ambient Gas on the Performance of Quantum-Dot Light-Emitting Diodes. ACS Appl. Mater. Interfaces 2016, 8, 11557-11563.

(56) Qian, L.; Zheng, Y.; Choudhury, K. R.; Bera, D.; So, F.; Xue, J.; Holloway, P. H. Electroluminescence from Light-Emitting Polymer/ZnO Nanoparticle Heterojunctions at Sub-Bandgap Voltages. Nano Today 2010, 5, 384-389. 
SYNOPSIS TOC

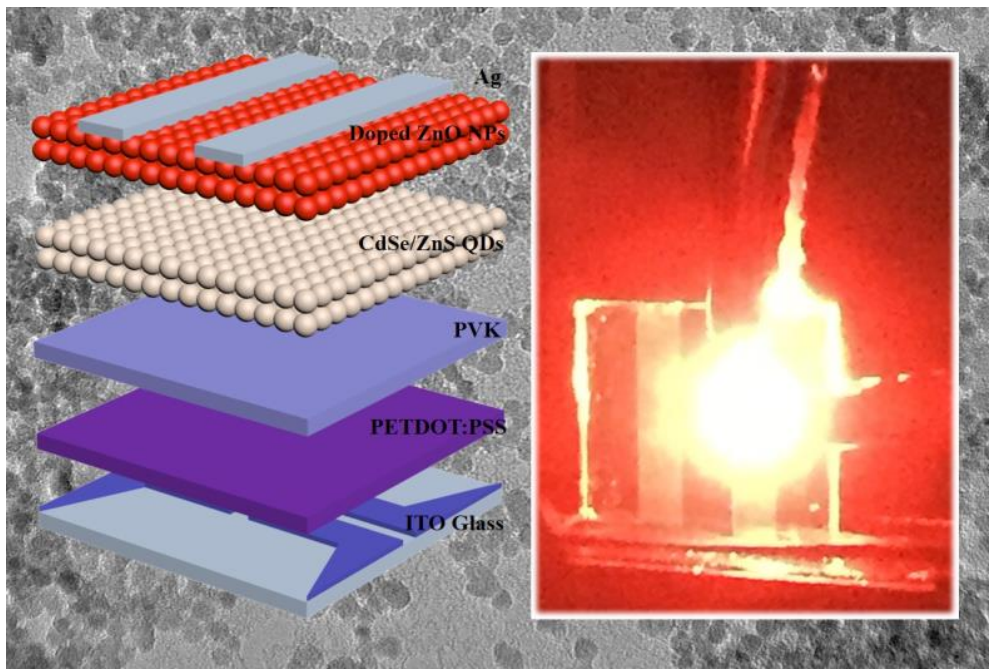

